\title{
New faint planetary nebulae from the DSS and SDSS
}

\author{
Matthias Kronberger ${ }^{1}$, George H. Jacoby ${ }^{2}$, Robin Ciardullo ${ }^{3}$, \\ Richard D. Crisp ${ }^{1}$, Orsola De Marco ${ }^{4}$, Dimitri Douchin ${ }^{4}$, \\ David J. Frew ${ }^{4}$, Dianne Harmer ${ }^{5}$, Mike Howell ${ }^{1}$, Steve B. Howell ${ }^{6}$, \\ Quentin A. Parker ${ }^{4,7}$, Dana Patchick ${ }^{1}$, Travis Rector ${ }^{8}$, Dave Riddle ${ }^{1}$ \\ and Philipp Teutsch ${ }^{1}$ \\ ${ }^{1}$ Deepskyhunters Collaboration \\ email: matthias.kronberger@cern.ch \\ ${ }^{2}$ GMTO Corporation, 813 Santa Barbara St, Pasadena, CA 91101 \\ email: gjacoby@gmto.org \\ ${ }^{3}$ Department of Astronomy and Astrophysics, Penn State University, PA 16802 \\ ${ }^{4}$ Department of Physics and Astronomy, Macquarie University, NSW 2109, Australia \\ ${ }^{5}$ NOAO, 950 N Cherry Ave, PO Box 26732, Tucson AZ 85726-6732 \\ ${ }^{6}$ NASA Ames Research Center, Moffett Field, CA 94035-1000 \\ ${ }^{7}$ Australian Astronomical Observatory, PO Box 296, Epping, NSW 1710, Australia \\ ${ }^{8}$ Department of Physics and Astronomy, University of Alaska Anchorage, AK 99508
}

\begin{abstract}
Having surveyed $\approx 10 \%$ of the sky, we have identified more than $130 \mathrm{PN}$ candidates by surveying multicolour Digitized Sky Survey (DSS), Sloan Digitized Sky Survey (SDSS), and combined [O III], $\mathrm{H} \alpha$ and $[\mathrm{S} \mathrm{II}]$ images. In a first imaging and spectroscopy campaign, 51 objects were identified as true and probable PNe. This work presents an additional 17 probable or possible PNe identified since that study. The majority of these candidates are situated at Galactic latitudes $|b|>5^{\circ}$, with the exception of seven objects located closer to the Galactic plane. Using the techniques described here that do not require any new survey data, we anticipate that many more PNe are waiting to be found, perhaps as many as 90 .
\end{abstract}

Keywords. planetary nebulae: general, surveys

Recent $\mathrm{H} \alpha$ surveys such as the AAO/UKST SuperCOSMOS $\mathrm{H} \alpha$ survey of the Southern Galactic plane (SHS: Parker et al. 2005) and the INT Photometric H $\alpha$ Survey of the Northern Milky Way (IPHAS: Drew et al. 2005) have been used to almost double the number of known Galactic planetary nebulae (PNe) (e.g. the Macquarie/AAO/ Strasbourg H $\alpha$ survey (MASH), Parker et al. 2006). However, both surveys cover only Milky Way regions close to the Galactic plane (SHS: $|b| \leqslant 10^{\circ}$; IPHAS: $|b| \leqslant 5^{\circ}$ ). Thus, it is likely that the current census of PNe is similarly incomplete beyond these limits.

The lack of high-resolution $\mathrm{H} \alpha$ or [O III] imagery in areas outside the SHS and IPHAS survey regions requires the use of other methods in order to identify new PNe. Possibilities are the visual inspection of multicolour Digitized Sky Survey (DSS) and Sloan Digitized Sky Survey (SDSS) images, and the survey for narrowband emitters on combined [O III], $\mathrm{H} \alpha$ and $[\mathrm{S} \mathrm{II}]$ images. Both techniques have been used in the search for unknown PNe by members of the Deep Sky Hunters (DSH) team.

Applying these survey methods to fields mostly complementary to those covered by SHS and IPHAS has led so far to the discovery of more than 130 PN candidates in a sky region of $\approx 4000^{\circ 2}$. In a first observing campaign, 76 candidates were imaged with the 
Table 1. New PN candidates

\begin{tabular}{|c|c|c|c|c|c|c|c|c|}
\hline Name & Other ID & R.A. J2000 & Dec. J2000 & $l\left({ }^{\circ}\right)$ & $b\left({ }^{\circ}\right)$ & Size $\left({ }^{\prime \prime}\right)$ & Status & Notes \\
\hline Kn 58 & & $02: 12: 27.9$ & $+47: 27: 10$ & 136.85 & -13.22 & $75 \times 52$ & prob. & \\
\hline $\mathrm{Kn} 60$ & & $07: 00: 06.7$ & $+12: 14: 40$ & 202.95 & +07.45 & $5 \times 5$ & prob. & \\
\hline Kn 41 & & $17: 11: 10.1$ & $-13: 57: 34$ & 008.35 & +14.83 & $32 \times 32$ & $\mathrm{PN}$ & \\
\hline Kn 42 & & $17: 38: 44.7$ & $-12: 57: 20$ & 012.87 & +09.69 & $11 \times 10$ & prob. & \\
\hline CGMW 4-2085 & & $18: 38: 07.9$ & $-31: 53: 00$ & 002.77 & -11.36 & $17 \times 15$ & $\mathrm{PN}$ & \\
\hline Kn 59 & & $18: 41: 41.9$ & $+65: 11: 58$ & 095.28 & +25.45 & $4 \times 4$ & prob. & 1 \\
\hline Te 6 & DSH J1909.9+1204 & $19: 09: 54.9$ & $+12: 04: 52$ & 045.77 & +01.46 & $13 \times 13$ & prob. & 2 \\
\hline Kn 61 & Soccer Ball Nebula & $19: 21: 38.9$ & $+38: 18: 57$ & 070.52 & +11.00 & $100 \times 92$ & prob. & 3 \\
\hline Kn 8 & DSH J1922.6+2433 & $19: 22: 39.4$ & $+24: 33: 02$ & 058.24 & +04.56 & $50 \times 44$ & poss. & \\
\hline Kn 56 & & $19: 37: 43.8$ & $-13: 51: 20$ & 025.49 & -16.41 & $40 \times 25$ & prob. & \\
\hline Kn 11 & DSH J1941.3+2430 & $19: 41: 19.1$ & $+24: 30: 53$ & 060.25 & +00.82 & $>6 \times 4$ & prob. & \\
\hline Kn 7 & DSH J1942.4+2145 & $19: 42: 26.1$ & $+21: 45: 23$ & 057.98 & -00.77 & $23 \times 15$ & prob. & 4 \\
\hline Kn 9 & DSH J1944.9+2245 & $19: 44: 59.1$ & $+22: 45: 49$ & 059.15 & -00.78 & $>330 \times 270$ & poss. & 5 \\
\hline Kn 14 & DSH J2009.7+2407 & $20: 09: 42.2$ & $+24: 07: 40$ & 063.26 & -04.91 & $16 \times 13$ & prob. & \\
\hline Kn 20 & DSH J2021.4+3005 & $20: 21: 26.5$ & $+30: 05: 39$ & 069.70 & -03.79 & $15 \times 15$ & prob. & \\
\hline $\mathrm{Kn} 48$ & & $20: 38: 09.2$ & $+61: 55: 03$ & 097.42 & +12.37 & $42 \times 36$ & prob. & \\
\hline Ri 2 & DSH J2050.0+3753 & $20: 50: 02.0$ & $+37: 53: 17$ & 079.50 & -03.87 & $38 \times 28$ & prob. & \\
\hline
\end{tabular}

Notes:

${ }^{1}$ Halo $228^{\prime \prime} \times 174^{\prime \prime} ;{ }^{2}=$ IPHAS J190954.7+120455, Viironen et al. $2009 ;{ }^{3}$ located in Kepler target field; ${ }^{4}=$ IPHASX J194226.2+214521, Viironen et al. $2009 ;{ }^{5}=$ WHI J1945+22, Whiting et al. 2007

WIYN $3.5 \mathrm{~m}$ and the OHP $1.2 \mathrm{~m}$ telescopes, and 60 of them were found to be probable or possible PNe, including $\approx 20 \%$ of spherical objects (Jacoby et al. 2010). Spectroscopic studies performed on 53 of these objects with the WIYN $3.5 \mathrm{~m}$ and SAAO $1.9 \mathrm{~m}$ telescopes confirmed 45 of them as PNe and identified one [WO] and at least two other emission line central stars (Frew et al. 2011, in prep.). Of the remaining seven targets without spectra, six have a high probability of being PNe based on their morphologies alone.

Since this first imaging and spectroscopy campaign, additional 17 objects from the candidate list have been identified as new possible and probable PNe. Table 1 lists the basic parameters of all objects. Objects described already in Kronberger et al. (2006) have their respective DSH IDs listed. Seven candidates were classified as probable or possible $\mathrm{PNe}$ according to their appearance on IPHAS $\mathrm{H} \alpha$ imagery. The remaining ten objects were imaged with the $2.1 \mathrm{~m}$ and $4 \mathrm{~m}$ KPNO telescopes and the WIYN $3.5 \mathrm{~m}$ telescope. The latter instrument was also used to confirm the objects Kn 41 and CGMW 4-2085 spectroscopically as PNe. One of the new candidates, the Soccer Ball Nebula Kn 61 , is located in the target field of the Kepler satellite, making it the sixth known PN in this sky region and the third one discovered in the framework of the DSH project after Pa 5 and PaTe 1 (Douchin et al. these proceedings).

From the number of objects identified so far, we anticipate that many more PNe can be identified with the described survey technique. By relating the number of new objects to the number of known PNe in the same region of sky, and extrapolating this to fields not yet surveyed, we estimate the number of additional detectable targets to $60 \pm 30$.

\section{References}

Douchin, D., Jacoby, G. H., De Marco, O., Howell, S., \& Kronberger, M. 2011, these proceedings Drew, J. E., Greimel, R., Irwin, M. J. et al. 2005, MNRAS, 362, 753

Jacoby, G. H., Kronberger, M., Patchick, D. et al. 2010, PASA, 27, 156

Kronberger, M., Teutsch, P., Alessi, B., Steine, M. et al. 2006, A\& A, 447, 921

Parker, Q. A., Phillipps, S., Pierce, M. J. et al. 2005, MNRAS, 362, 689

Parker, Q. A., Acker, A., Frew, D. J., Hartley, M., \& Peyaud, A. E. J. et al. 2006, MNRAS, 373, 79

Viironen, K., Greimel, R., Corradi, R. L. M. et al. 2009, A\& A, 504, 291

Whiting, A. B., Hau, G. K. T., Irwin, M., \& Verdugo, M. 2007, AJ, 133, 715 